\title{
Evaluación de la sostenibilidad de cultivos de papa (páramo de Gámeza, Boyacá, sector Daita, Colombia)
}

\author{
Assessment of the Sustainability of Potato Crops (Moorland Gámeza, Boyacá, Daita sector, Colombia)
}

\author{
Cristian Andrés Sanabria Hurtado ${ }^{a c}$, Inés Andrea Sanabria Totaitive ${ }^{a d}$, Rafael Eduardo Sánchez Cuervo ${ }^{\text {be }}$ \\ ${ }^{a}$ Grupo de Estudios en Etología, Ecología, Educación y Conservación (GECOS), Licenciatura en Ciencias Naturales y Educación Ambiental, \\ Facultad de Ciencias de la Educación, Universidad Pedagógica y Tecnológica de Colombia, Tunja, Colombia

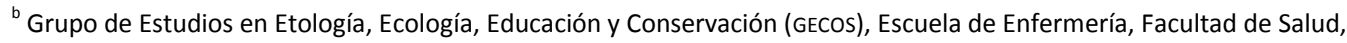 \\ Universidad Pedagógica y Tecnológica de Colombia, Tunja, Colombia \\ ${ }^{c}$ cristian.sanabria@uptc.edu.co | https://orcid.org/0000-0003-0608-2425 \\ ${ }^{d}$ inesandrea.sanabria@uptc.edu.co | https://orcid.org/0000-0001-7791-3738 \\ e rafael.sanchezcuervo@uptc.edu.co | https://orcid.org/0000-0001-6911-6624
}

Citation: Sanabria-Hurtado, C. A., Sanabria-Totaitive, I. A. y SánchezCuervo, R. E. (2022). Evaluación de la sostenibilidad de cultivos de papa (páramo de Gámeza, Boyacá, sector Daita, Colombia). Mutis, 12(1). https://doi.org/10.21789/22561498.1769

Recibido: 10 de junio de 2021 Aceptado: 15 de agosto de 2021

Copyright: () 2021 por los autores. Licenciado para Mutis. Este artículo es un artículo de acceso abierto distribuido bajo los términos y condiciones de la licencia Creative Commons Attribution (https:// creativecommons.org/licenses/by/ 4.0/).

\section{RESUMEN}

Este trabajo muestra el análisis y posterior evaluación de la sostenibilidad en tres sistemas de producción de papa en el municipio de Gámeza (Boyacá, Colombia) durante 2019. A nivel metodológico, la información fue registrada a partir de mediciones hechas en campo, entrevistas a los propietarios de los cultivos y observación participante, con el fin de identificar puntos críticos usados para construir 11 indicadores integrados en 36 índices. Adicionalmente, se efectuó una revisión bibliográfica y consulta a los agricultores para la obtención de valores óptimos por cada índice, y a partir de la estandarización de los datos a porcentajes se realizaron gráficas de ameba. Los resultados muestran que existen diferencias y similitudes en los agroecosistemas, en razón de las prácticas agrícolas y la gestión ambiental. La principal vulnerabilidad de los tres sistemas se registró en los índices que miden la dependencia de insumos externos, las oportunidades de capacitación y la composición de nutrientes en el suelo. Se concluye que los tres sistemas no son sustentables en todos los indicadores. Por consiguiente, acciones como capacitaciones técnicas, reforestación de flora nativa, integración colectiva de los agricultores, estudios de tipo biológico y social y acuerdos colectivos entre entidades gubernamentales y agricultores son necesarias para dar lugar a cultivos de papa sostenibles.

Palabras clave: valoración de la sostenibilidad, agricultura, medioambiente, desarrollo agrícola, conservación ambiental.

\section{ABSTRACT}

This work shows the analysis and subsequent evaluation of the sustainability of three potato production systems in the municipality of Gámeza (Boyacá, Colombia) during 2019. Methodologically, the information was gathered through field measurements, interviews with crop owners, and participant observation, with the purpose of finding critical points that were later used to build 11 indicators integrated in 36 indexes. Additionally, a bibliographic review was carried out and farmers were consulted to obtain optimal values for each index. Radar charts were made based on the standardization of the data into percentages. The results show differences and similarities in the agroecosystems studied due to the agricultural practices and environmental 
management approaches deployed. The main vulnerability of the three systems was recorded in the indices that measure dependence on external inputs, training opportunities, and soil nutrient composition. It is concluded that the three systems are not sustainable in all indicators. Therefore, actions such as technical training, reforestation of native flora, collective integration of farmers, biological and social studies, and collective agreements between governmental entities and farmers become necessary to initiate sustainable potato crops.

Keywords: Sustainability assessment, agriculture, environment, agricultural development, environmental conservation.

\section{INTRODUCCIÓN}

La agricultura campesina de los Andes es usada como fuente de sustento para las familias de la región, quienes a partir de técnicas agrícolas específicas han desarrollado sistemas productivos, principalmente de papa (Solanum tuberosum) (Insuasty-Córdoba et al., 2020). Este alimento es producido en todo el mundo y posee una marcada importancia económica y social debido a su valor nutricional, otorgado por sus altos porcentajes de almidón y antioxidantes, como polifenoles, minerales esenciales, aminoácidos y vitaminas B6, B3 y C (Van-Dingenen et al., 2019). Asimismo, este producto permite generar empleo $\mathrm{y}$, con el manejo técnico adecuado, puede constituir producciones viables y sustentables, aportando rentabilidad económica para los campesinos (Incacari et al., 2019). En el mundo, la agricultura proporciona el $40 \%$ de empleos y suministra el $70 \%$ de alimentos, aunque tan solo representa 3,9\% del producto interno bruto global (PIB) (Calleros-Islas, 2019), situación que apunta a posibles niveles de pobreza en los agricultores.

A nivel ambiental, la producción de papa conlleva actividades como quemas, tala de bosques nativos y manejo agronómico a partir del uso de fertilizantes químicos (Erbaugh et al., 2019). En consecuencia, aparecen condiciones ambientales negativas como sequedad del suelo, disminución de la capa freática, pérdida de biodiversidad, mayor frecuencia de eventos hidrometereológicos extremos y deterioro de la salud humana (Acevedo-Osorio et al., 2017; Tang et al., 2019). La mayor producción de papa se realiza en páramos, usualmente con prácticas insostenibles que ponen en riesgo el ambiente y su permanencia como actividad económica y social a corto o largo plazo. En ese sentido, resulta importante fomentar sistemas agrícolas que satisfagan las necesidades alimentarias sin comprometer el medio ambiente, la economía y los espacios socioculturales para las generaciones presentes y futuras (Bonisoli et al., 2018; Calleros-Islas, 2019; Cruz et al., 2016).

Al respecto, se han realizado diversas evaluaciones de la sustentabilidad con métodos multicriterio, a partir del diseño de indicadores a escala global y local (Acevedo-Osorio et al., 2017; Barrezueta-Unda \& Paz-González, 2018; Márquez et al., 2020). Estos indicadores son medidas cuantitativas que permiten mejorar la toma de decisiones, efectuando análisis robustos y aplicables en respuesta a los desafíos globales, desde la lectura del contexto local, con la participación de comunidades (Bonisoli et al., 2018; Calleros-Islas, 2019). Por consiguiente, el objetivo de este trabajo fue evaluar, con ayuda de indicadores, las dimensiones ambiental, técnica y económica y el nivel de sustentabilidad de tres fincas dedicadas a la producción de papa en zona de páramo, en la vereda Daita del municipio de Gámeza, Boyacá (Colombia), durante el ciclo de producción de 2019. 


\section{MATERIALES Y MÉTODOS}

\section{Área de estudio}

El estudio se realizó en la vereda Daita, ubicada en el municipio de Gámeza (departamento de Boyacá, Colombia), en un rango altitudinal que comprende desde los 3.100 hasta los 3.600 m s. n. m. y una extensión de 15.774 km² (figura 1). Las características del paisaje son propias de ecosistemas de páramo, subpáramo y bosque altoandino, con presencia de relictos de bosque nativo y adecuación de terrenos para uso agropecuario. Durante el año la región cuenta con un clima bimodal de temporada seca y temporada de lluvias, con temperaturas variadas comprendidas entre 2 y 18 ${ }^{\circ} \mathrm{C}$. El estudio en esta área constituye un análisis único de elementos representados en diversidad de flora, uso de recursos naturales y actividades productivas relacionadas con el cultivo de papa.

Figura 1. Sitio de estudio de las fincas: Los Chorros, Buenavista y La Laguna, ubicadas en el sector Daita del municipio de Gámeza.

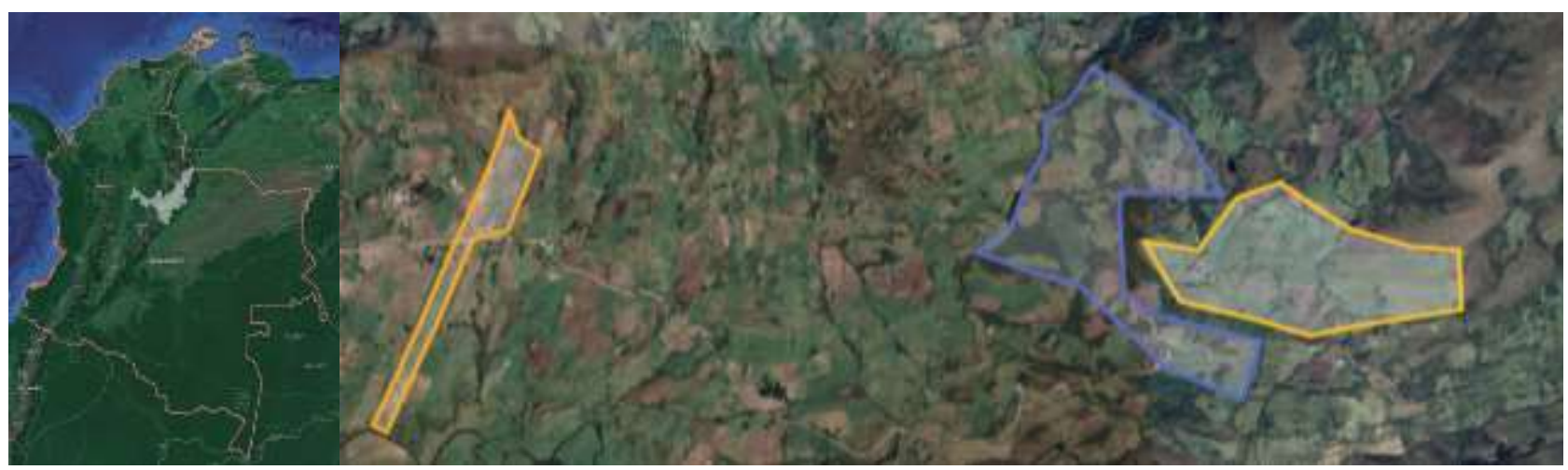

Fuente: adaptado de Google Earth (2021).

\section{Métodos y población de estudio}

La investigación se efectuó usando métodos cualitativos y se apoyó en resultados cuantitativos, siguiendo un alcance exploratorio y descriptivo. Esta se llevó a cabo entre de diciembre de 2018 y agosto de 2019. La población estuvo conformada por 18 fincas con reportes de siembra de papa, de las cuales se eligieron tres (Los Chorros, Buenavista y La Laguna), respondiendo a los siguientes criterios de inclusión: (i) registro contable de ingresos y costos por ciclo productivo; (ii) disponibilidad para brindar información, aceptando la participación en el trabajo previa firma de consentimiento informado; (iii) siembra mayor a ochenta bultos por ciclo productivo; (iv) tener como principal fuente de economía familiar la producción de papa.

\section{Recolección de la información, análisis y procesamiento}

La evaluación de la sustentabilidad se realizó siguiendo lo estipulado en el método MESMIS (Masera et al., 2000), de acuerdo con los seis pasos que se describen a continuación:

(i) Caracterización de los sistemas a evaluar: se buscó identificar elementos que permitieran describir las tres fincas seleccionadas desde las dimensiones social, ambiental y económica. Esto se hizo usando matrices DOFA y entrevistas con preguntas basadas en criterios de diagnóstico para 
los agricultores. Adicionalmente, se registraron datos climatológicos con la adecuación de un termómetro y un pluviómetro que fueron ubicados en mediaciones a los tres sistemas productivos. Finalmente, se llevaron a cabo cuatro salidas de campo en los meses de marzo y junio para caracterizar la flora del lugar, trazando tres transectos de $50 \mathrm{~m}$ de largo por $1 \mathrm{~m}$ de ancho, en cuatro relictos de bosque nativo cercanos a los terrenos cultivados.

(ii) Identificación de los puntos críticos del sistema: con los resultados obtenidos en el paso (i), se identificaron aspectos que ponen en riesgo la permanencia de los cultivos, por vulnerabilidades en lo técnico, lo ambiental y lo socioeconómico.

(iii) Selección de los indicadores con sus respectivos índices: estos fueron construidos a partir de lo compilado en referencias bibliográficas, así como de los puntos críticos identificados y de los atributos correspondientes a productividad, estabilidad, confiabilidad, resiliencia, adaptabilidad, autodependencia y equidad (Masera et al., 2000).

(iv) Medición y monitoreo de cada uno de los indicadores: para la dimensión económica y social la información fue registrada con preguntas a los productores y observación participante durante toda la fenología del ciclo vegetativo y productivo de la papa. En cuanto a la dimensión ambiental, se realizaron seis salidas de campo. Con ayuda de GPS se calcularon en $\mathrm{m}^{2}$ las áreas totales, conservadas y destinadas para cultivar en cada finca. Igualmente se realizó un análisis de suelos para evaluar parámetros químicos, trazando en zigzag un transecto con cincos puntos en cada unidad de producción. En cada punto se localizó una subparcela con un área de $3 \times 3 \mathrm{~m}$, en la cual se recolectaron dos submuestras de suelo a profundidades de 0 $20 \mathrm{~cm}$, para conformar una muestra compuesta por punto y luego por finca (Bravo-Medina et al., 2017). Además, se tomó una muestra de suelo en bosque nativo, buscando la mínima fuente de contaminación. Todas las muestras fueron llevadas para su análisis al laboratorio Agrosoil, en donde se les realizó un análisis fisicoquímico (tabla 1).

Tabla 1. Parámetros fisicoquímicos y su respectivo método de medición

\begin{tabular}{|c|c|}
\hline Parámetro & Método \\
\hline Contenido de $\mathrm{Ca}$ (meq/100 g de suelo) & \multirow{3}{*}{ Absorción atómica, extracción con acetato de amonio } \\
\hline Contenido de $\mathrm{Mg}$ (meq/100 g de suelo) & \\
\hline Contenido de $\mathrm{K}$ (meq/100 g de suelo) & \\
\hline $\mathrm{pH}$ & Con potenciométrico, usando una relación suelo: agua 1:1 \\
\hline Materia orgánica (\%) & Walkley Black \\
\hline Contenido de $\mathrm{N} \mathrm{mg/kg}$ & Cálculo matemático \\
\hline Contenido de $\mathrm{S} \mathrm{mg} / \mathrm{k}$ & Por turbidimétrico, mediante extracción de fosfato monobásico de calcio $0.008 \mathrm{M}$ \\
\hline Contenido de $\mathrm{P}$ (mg/kg) & Por colorimétrico, Bray II \\
\hline Contenido de $\mathrm{Mn}(\mathrm{mg} / \mathrm{Kg})$ & \multirow{3}{*}{ Absorción atómica, extracción con DTPA } \\
\hline Contenido de $\mathrm{Zn}(\mathrm{mg} / \mathrm{Kg})$ & \\
\hline Contenido de $\mathrm{Cu}(\mathrm{mg} / \mathrm{kg})$ & \\
\hline
\end{tabular}

Fuente: elaboración propia. 
(v) Integración de resultados: la diversidad de flora se graficó en una curva de rango-abundancia, con transformación logarítmica de base 10 (Feinsinger, 2003), usando el programa estadístico R versión 3.6.1 y el paquete ggplot2. Los resultados obtenidos en los índices fueron estandarizados a porcentajes, de acuerdo con los valores máximos del parámetro consultado en la literatura, por consenso de agricultores o según lo dicho por un experto (campesino con experiencia). Para los indicadores cualitativos, se usó la métrica Sí $=100 \%$, No $=0 \%$; en los categóricos, el porcentaje fue dividido equitativamente entre el número de categorías (muy bajo $=25 \%$, bajo $=50 \%$, alto $=75 \%$, muy alto $=100 \%$ ). A partir de este ajuste y de la estandarización a porcentaje, para mostrar el nivel de distancia porcentual de cada indicador para los tres cultivos de papa, se elaboraron gráficos de ameba (Montes-Pérez et al., 2016). Adicionalmente, con el fin de determinar si existe diferencia significativa en cuanto a los valores obtenidos en el conjunto de índices por cada dimensión entre las tres zonas, teniendo en cuenta la no normalidad de los datos evaluada mediante la prueba de Shapiro-Willk, se realizó una prueba estadística no paramétrica Kruskal-Wallis, con un valor de probabilidad de 0,05. Los análisis se realizaron en el software libre $R$ versión 3.6.1. y con Microsoft Excel.

(vi) Conclusiones y recomendaciones sobre los sistemas de manejo: a partir del análisis de los resultados se elaboraron las conclusiones y recomendaciones, en lo general y particular, para cada productor.

\section{RESULTADOS Y DISCUSIÓN}

En la vereda Daita habitan 18 familias dedicadas a la producción y comercialización de papa (Solanum tuberosum, variedad tocarreña o sabanera), leche y, en menor cuantía, hortalizas. La flora que aún se conserva, con mayor presencia de especies como Ageratina boyacensis (figura 2), se encuentra en pequeños relictos de bosque, como sucede en la mayor parte de las regiones con similares paisajes en el territorio colombiano (Avellaneda-Torres et al., 2015).

Según los entrevistados, la disminución en la vegetación ha estado acompañada de cambios en los niveles de precipitación y temperatura, provocando variaciones en la intensidad de heladas, así como de los periodos lluviosos. Para el caso del ciclo productivo evaluado, se presentó un período seco en los meses de diciembre de 2018 y enero y febrero de 2019, con precipitaciones promedio de 3 $\mathrm{mm}$ y temperaturas máxima de $24{ }^{\circ} \mathrm{C}$ y mínima de $3{ }^{\circ} \mathrm{C}$, y otro período lluvioso durante los meses de mayo, junio, julio y agosto de 2019 , con precipitaciones promedio de $17 \mathrm{~mm}$ y temperaturas máxima de $14^{\circ} \mathrm{C}$ y mínima de $4^{\circ} \mathrm{C}$. Estas condiciones favorecen la adecuación de cultivos $y$, de acuerdo con los registros de producción y relatos de los agricultores, corresponden a variaciones de humedad óptimas, por cuanto se asegura adecuada germinación de la semilla y posterior desarrollo de la planta. 
Figura 2. Abundancia de flora del lugar donde se ubican los sistemas productivos

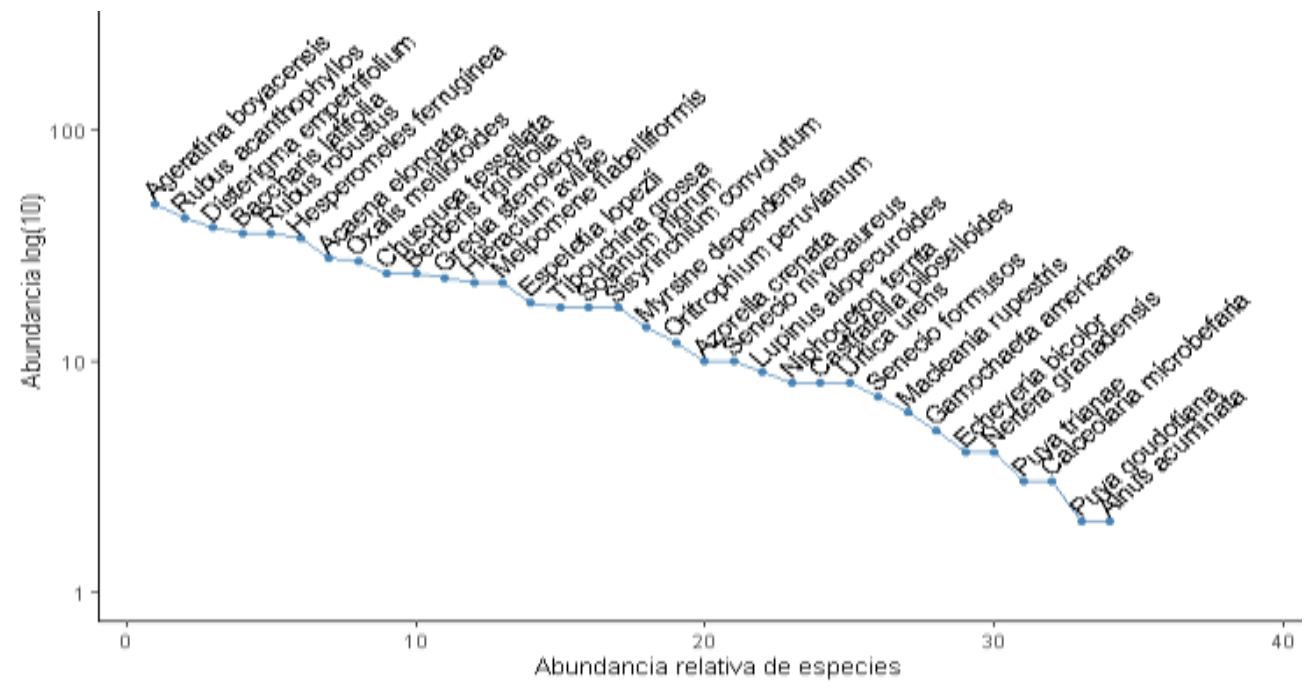

Fuente: elaboración propia.

En cuanto a la valoración de la sustentabilidad en las fincas La Laguna, Buenavista y Los Chorros, se identificaron 11 indicadores (tabla 2) agrupados en 36 índices. Los atributos y criterios de diagnóstico empleados permiten encontrar semejanzas con lo ya reportado en los trabajos de Acevedo-Osorio et al. (2017), Barrezueta-Unda y Paz-González (2018), Cárdenas y Acevedo (2015) e Incacari et al. (2019), siendo la rentabilidad, la eficiencia y la autodependencia los factores con mayor influencia en la viabilidad de los cultivos.

Tabla 2. Indicadores seleccionados: dimensión ambiental (A), dimensión económica (E), dimensión social (S)

\begin{tabular}{|c|c|c|c|}
\hline Atributo & Criterio de diagnóstico & Indicadores & Área de evaluación \\
\hline \multirow[t]{2}{*}{ Productividad } & Rentabilidad & Beneficio/costo & $\mathrm{E}$ \\
\hline & Eficiencia & Eficiencia técnica & $\mathrm{E}$ \\
\hline \multirow{6}{*}{ Estabilidad resiliencia } & Fragilidad del sistema & $\begin{array}{l}\text { Prácticas para el manejo de plagas } \\
\text { y enfermedades }\end{array}$ & A \\
\hline & \multirow{3}{*}{ Conservación de recursos } & Calidad de suelo & A \\
\hline & & Calidad del agua & A \\
\hline & & Estado de la biodiversidad & A \\
\hline & \multirow{2}{*}{ Vulnerabilidad económica } & Ingresos diversificados & $\mathrm{E}$ \\
\hline & & Disponibilidad de mano de obra & $S$ \\
\hline Adaptabilidad & Capacidad de cambio e innovación & $\begin{array}{l}\text { Capacidad de generación y cono- } \\
\text { cimientos }\end{array}$ & $S$ \\
\hline Equidad & $\begin{array}{l}\text { Distribución de beneficios y toma de } \\
\text { decisiones }\end{array}$ & Beneficios del sistema & $\mathrm{S}$ \\
\hline Auto dependencia & Autosuficiencia & Dependencia externa & $\begin{array}{l}A \\
S\end{array}$ \\
\hline
\end{tabular}

Fuente: adaptado de Incacari et al. (2019), Fonseca-Carreño et al. (2015), Barrezueta-Unda y Paz-González (2018), Bravo-Medina et al. (2017) y Motta-Delgado et al. (2019). 
Estadísticamente, no se encontró diferencia significativa entre los valores obtenidos por cada conjunto de índices por dimensión y fincas, con $p$-valores superiores a 0,05 . El $p$-valor más bajo se registró en la dimensión social entre las fincas Los Chorros y La Laguna $(p=0,09)$, mientras que en la dimensión económica se registraron los valores más elevados $(p=0,44)$. Estos datos indican la similitud en los métodos usados para la producción de papa, los cuales se ligan a prácticas tradicionales y uso de agroquímicos (Avellaneda-Torres et al., 2015).

Para el caso de la dimensión ambiental, se encontraron similitudes entre los agrosistemas. La primera es el uso de insumos externos y agroquímicos para aumentar la producción. De igual manera, existe poca diversificación de cultivos, si bien se registran sembradíos de pastos (avena y raigrás), aunque los cultivos de papa en un mismo terreno suelen ser consecutivos por tres o más años (tabla 3 ).

Tabla 3. Resultado de los indicadores de la dimensión ambiental

\begin{tabular}{|c|c|c|c|c|c|c|}
\hline Indicador & Índice & La Laguna & $\begin{array}{c}\text { Los } \\
\text { Chorros }\end{array}$ & Buena vista & Óptimo & Criterio óptimo \\
\hline \multirow{2}{*}{$\begin{array}{l}\text { Prácticas para } \\
\text { el manejo de } \\
\text { plagas y } \\
\text { enfermedades }\end{array}$} & $\begin{array}{l}\text { Empleo de productos } \\
\text { biológicos en el control } \\
\text { de daños (\%) }\end{array}$ & 0 & 0 & 0 & 100 & Avellaneda-Torres et al. (2015) \\
\hline & $\begin{array}{c}\text { Cantidad de pesticidas y } \\
\text { herbicidas usados en el } \\
\text { cultivo }(\%)\end{array}$ & 75 & 25 & 25 & 25 & Avellaneda-Torres et al. (2018) \\
\hline \multirow{8}{*}{$\begin{array}{l}\text { Calidad de } \\
\text { suelo }\end{array}$} & $\begin{array}{c}\text { Diversificación de } \\
\text { cultivos }\end{array}$ & 50 & 25 & 25 & 25 & Calleros-Islas (2019) \\
\hline & Materia orgánica (\%) & 11,42 & 12,7 & 12,4 & 17,7 & \multirow{7}{*}{ Muestra zona no intervenida } \\
\hline & Concentración N (\%) & 0,57 & 0,63 & 0,64 & 0,89 & \\
\hline & $\begin{array}{l}\text { Contenido de } \mathrm{P} \\
\text { (mg/kg de suelo) }\end{array}$ & 96,74 & 28,34 & 33,22 & 15,95 & \\
\hline & $\begin{array}{c}\text { Contenido de Ca } \\
\text { (meq/100 g de suelo) }\end{array}$ & 12,31 & 2,89 & 3,22 & 4,51 & \\
\hline & $\begin{array}{c}\text { Contenido de Mg } \\
\text { (meq/100 g de suelo) }\end{array}$ & 0,67 & 0,62 & 0,67 & 0,92 & \\
\hline & $\begin{array}{c}\text { Contenido de } \mathrm{K} \\
\text { (meq/100 g de suelo) }\end{array}$ & 0,45 & 0,69 & 0,74 & 0,51 & \\
\hline & $\mathrm{pH}$ & 5,49 & 4,48 & 4,58 & 4,44 & \\
\hline \multirow{2}{*}{$\begin{array}{l}\text { Calidad del } \\
\text { agua }\end{array}$} & $\begin{array}{c}\text { Uso de prácticas para } \\
\text { mitigar la } \\
\text { contaminación de agua }\end{array}$ & 50 & 50 & 50 & 100 & Criterio de agricultores \\
\hline & $\begin{array}{c}\text { Humedales protegidos } \\
(\%)\end{array}$ & 6 & 20 & 35 & 75 & Avellaneda-Torres et al. 2018) \\
\hline $\begin{array}{l}\text { Estado de la } \\
\text { biodiversidad }\end{array}$ & $\begin{array}{l}\text { Área de zonas protegi- } \\
\text { das con bosque } \\
\text { natural/área total de la } \\
\text { finca }\left(\mathrm{m}^{2}\right)\end{array}$ & $\begin{array}{l}27.007 / \\
135.582\end{array}$ & $\begin{array}{l}23.321 / \\
252.162\end{array}$ & $\begin{array}{l}223.566 / \\
438.949\end{array}$ & $\begin{array}{c}3.000 / \\
9.000\end{array}$ & $\begin{array}{c}\text { Valor por consenso de } \\
\text { agricultores }\end{array}$ \\
\hline $\begin{array}{l}\text { Dependencia } \\
\text { externa }\end{array}$ & $\begin{array}{c}\text { Insumos } \\
\text { externos/unidad de } \\
\text { producto }\end{array}$ & 25 & 25 & 25 & 100 & Criterio de agricultores \\
\hline
\end{tabular}

Fuente: elaboración propia. 
En la figura 3, los resultados obtenidos evidencian transformaciones ecológicas atribuibles a actividades agropecuarias en hábitats naturales, aspecto ya señalado en estudios como el de Avellaneda-Torres et al. (2018), Calleros-Islas (2019), Fonseca-Carreño et al. (2015) y Gitari et al. (2018). En consecuencia, se incrementa la pérdida de especies y disminuye la capacidad de resiliencia y estabilidad biológica, afectando el funcionamiento y potencial para que los ecosistemas respondan y se adapten a cambios en cuanto a condiciones físicas y bióticas generadas por la agricultura (Antonini \& Larrinaga, 2017).

Figura 3. Índices de la dimensión ambiental: diversificación de cultivos (DC), empleo de productos biológicos en el control de daños (EP), humedales protegidos (HU), insumos externos/unidad de producto (IE), materia orgánica (MO), pH del suelo (ph), uso de prácticas para mitigar la contaminación de agua (UP), área de zonas protegidas con bosque natural (AP), contenido de $\mathrm{Ca}$ (CCa), contenido de $\mathrm{K}$ (CK), contenido de Mg (CMg), contenido de $\mathrm{N}$ (CN), contenido de $\mathrm{P}(\mathrm{CP})$, cantidad de pesticidas y herbicidas usados en el cultivo (CPH).

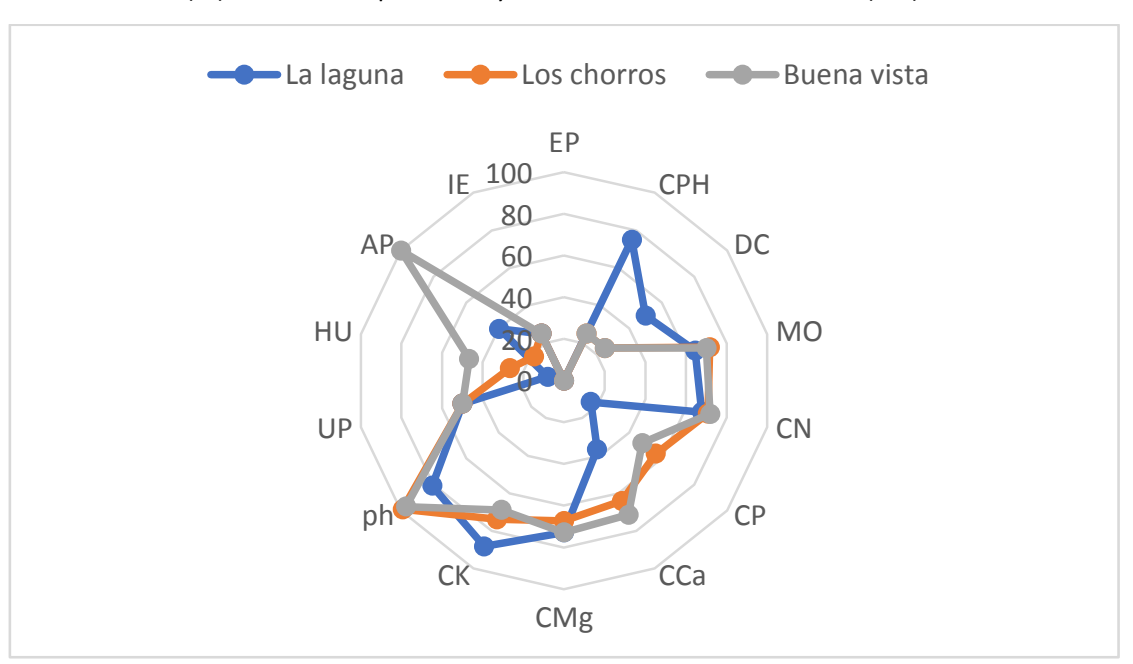

Fuente: elaboración propia.

En relación con los valores obtenidos de suelos no intervenidos por agricultura, se evidencian bajos contenidos de materia orgánica y alteración en la concentración de bioelementos químicos, indicadores de continuas transformaciones del ambiente natural a causa de la agricultura alto-andina (Acevedo-Osorio et al., 2017). Al respecto, Avellaneda-Torres et al. (2018) señalan que la disminución de materia orgánica se debe a la mayor mineralización de residuos de los cultivos, la alteración de los agregados del suelo y el aumento de la aireación. A su vez, Álvarez-Yela et al. (2017) mencionan que las actividades agrícolas conducen a un cambio en la estructura de las comunidades microbianas, afectando de manera indirecta la regulación del ciclo de nutrientes, principalmente nitrógeno y carbono.

En este sentido, Van-Dingenen et al. (2019) describen que la razón por la cual los agricultores aplican grandes cantidades de fertilizantes es por su efecto en el crecimiento de las plantas. Sin embargo, para el caso del nitrógeno (N), solo el $50 \%$ es realmente utilizado por el cultivo; el excedente se filtra. Igualmente, la ausencia de fijadores de nutrientes provoca concentraciones excesivas de algunos elementos, causando problemas ambientales y de salud humana (Álvarez-Yela et al., 2017).

Ante esta situación es necesario garantizar condiciones técnicas, consolidadas a partir de estudios locales, capaces de reducir daños ambientales y contribuir con el bienestar de las comunidades campesinas. Una iniciativa al respecto 
es la reforestación con especies nativas en pequeñas áreas de cada finca, dando lugar a corredores biológicos y al aprovechamiento de servicios ecosistémicos ofrecidos por plantas propias de la zona alto-andina, tales como Bocconia frutescens, Gleditsia triacanthos, Smallanthus pyramidalis y Alnus acuminata, las cuales permiten la activación biológica del suelo, el ciclo de nutrientes y la acción natural de predadores sobre parásitos (Acevedo-Osorio et al., 2017).

En cuanto a la dimensión social (figura 4), como ha sido previamente reportado por Avellaneda-Torres et al. (2014), hay escasa participación en capacitaciones, además de que el uso de abonos y fungicidas se basa en las indicaciones del vendedor, y aunque se altere la composición del suelo y exista riesgo por deterioro de la salud, los agroquímicos han proporcionado soluciones al rendimiento productivo en corto tiempo. Por otro lado, de las tres fincas, en Los Chorros los miembros de la familia son quienes participan en las labores de los cultivos, contrario a lo que sucede en Buenavista y La Laguna, donde la integración social se basa en la ayuda comunitaria entre agricultores.

En lo que se refiere a los índices de tenencia de la tierra y el porcentaje de necesidades básicas cubiertas por los cultivos, las tres familias alcanzan su seguridad alimentaria, propiciando espacios de bienestar social y equidad, con el agregado de generar empleo. Esto ha permitido que los productores de las fincas La Laguna y Los Chorros dispongan de terreno propio para las actividades agropecuarias, encontrando la posibilidad de diversificar los cultivos y ampliar sus fuentes de ingreso.

Figura 4. Índices de la dimensión social: cambios en la salud de la familia (RAC), tipo y frecuencia de capacitación (TFC), tenencia de la tierra (TT), tipo de vivienda del agricultor (TV), personas para realizar las labores de producción (FPF), integración social (IS), \% de necesidades básicas cubiertas con la producción de papa (PNB).

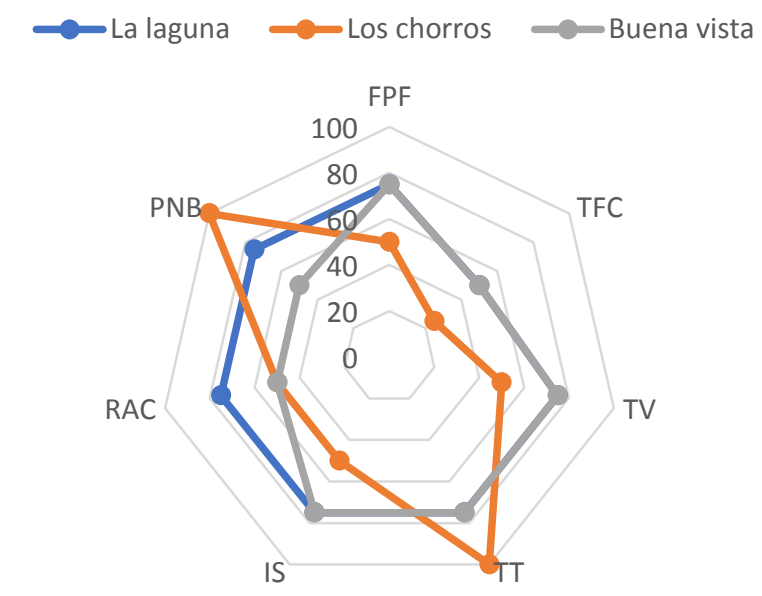

Fuente: elaboración propia.

Para la dimensión económica, aunque en la tabla 4 se muestran balances positivos en cuanto a ganancias netas, la eficiencia económica de los cultivos de papa no siempre es positiva. El índice que más influye es la variedad de los precios, los cuales, para 2019, no superaron una diferencia de COP 100.000, moneda corriente. Sin embargo, los agricultores registran variaciones que superan COP 150.000, de ahí que en algunos años el ingreso haya sido menor que los costos. No obstante, una forma de alcanzar buenos precios por ventas es realizar el cultivo en mayo y los primeros días 
del mes de junio, por lo que es necesario iniciar las siembras en octubre y noviembre, asumiendo el riesgo de pérdidas por heladas e intensidad del verano.

Un ejemplo de lo anterior es la finca La Laguna, que asume dicho riesgo al disponer de sistemas de regadío y conocimiento tradicional para combatir heladas con hogueras en la madrugada. Aunque como resultado obtiene bajo rendimiento en cuanto a producción, alcanza precios por ventas superiores a la inversión, sumado a que no expone sus cultivos a las fuertes lluvias que inician en los meses de abril. En las fincas Buenavista y Los Chorros los cultivos comienzan desde los meses de diciembre, sin uso de regadíos y mejor producción; sin embargo, los costos aumentan al usar en gran medida fungicidas para combatir los daños por gota a causa de las fuertes lluvias, lo cual, junto con la variación en los precios del mercado, genera el riesgo de disminuir la ganancia monetaria, en concordancia con los reportes de Fonseca-Carreño et al. (2015).

Tabla 4. Resultado de indicadores de dimensión económica

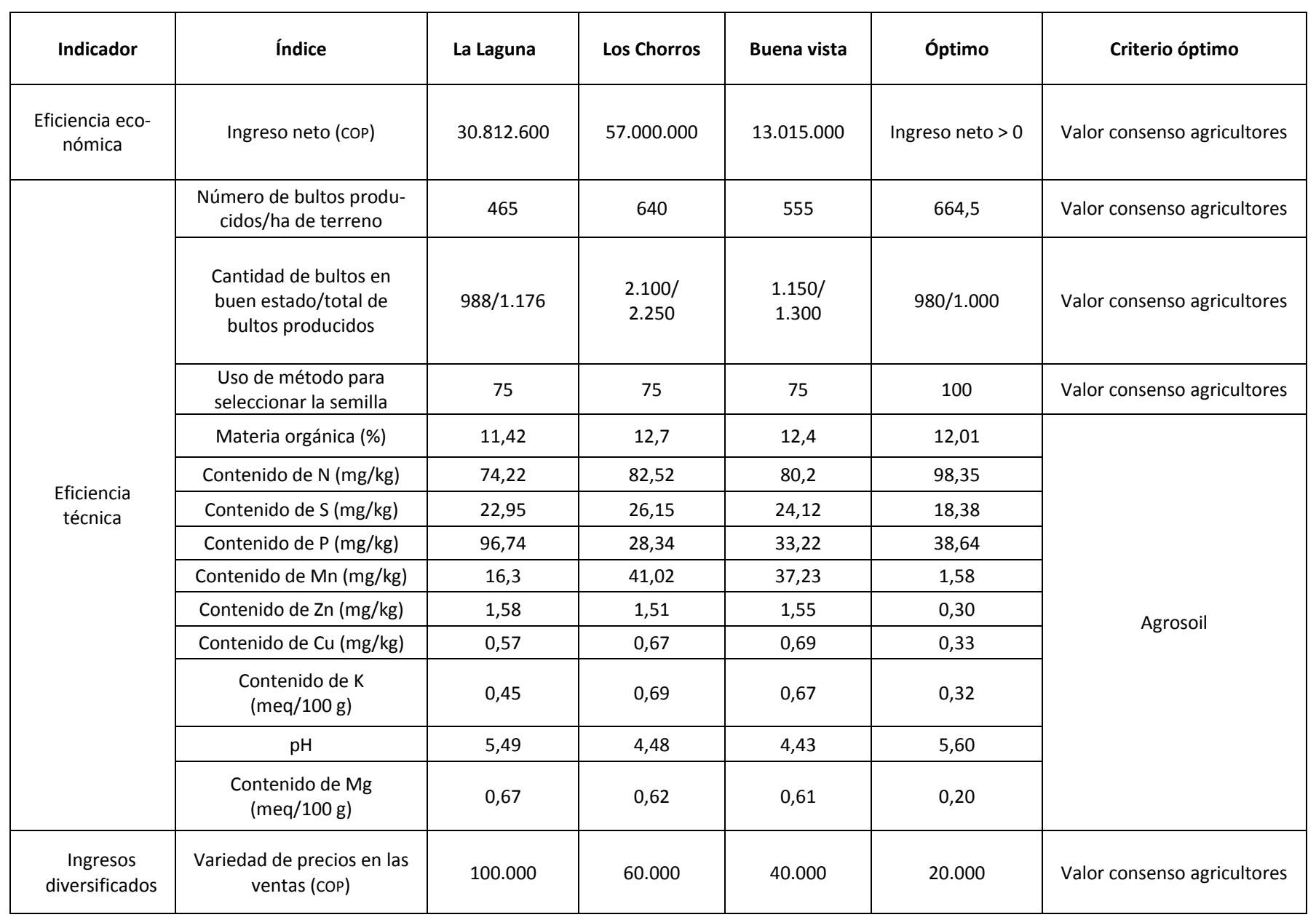

Fuente: elaboración propia. 
En la figura 5 se muestra el radar comparativo de cada uno de los índices seleccionados para evaluar los indicadores de la dimensión económica. Se observa que el mejor comportamiento para índices como $\mathrm{pH}$, contenidos de $\mathrm{K}$ y de $\mathrm{S}$ lo tiene la finca La Laguna, mientras que Buenavista presentó menor variación de precios. Por otra parte, Los Chorros produce 640 bultos/ha y 2.100 en buen estado, de 2.250 obtenidos en total, superando a las demás fincas evaluadas. Esto supone una mejor optimización de recursos y manejo técnico para obtener rendimientos satisfactorios, que le permiten hacer rentable su cultivo.

Figura 5. Índices de la dimensión económica: contenido de S (CS), contenido de Zn (Czn), ingreso neto (INE), materia orgánica (MO), número de bultos producidos/ha de terreno (NBP), pH del suelo (ph), uso de método para seleccionar la semilla (UMS), variedad de precios en las ventas (VP), cantidad de bultos en buen estado/total de bultos producidos ( contenido de $\mathrm{N}(\mathrm{CN})$, contenido de $\mathrm{P}(\mathrm{CP})$.

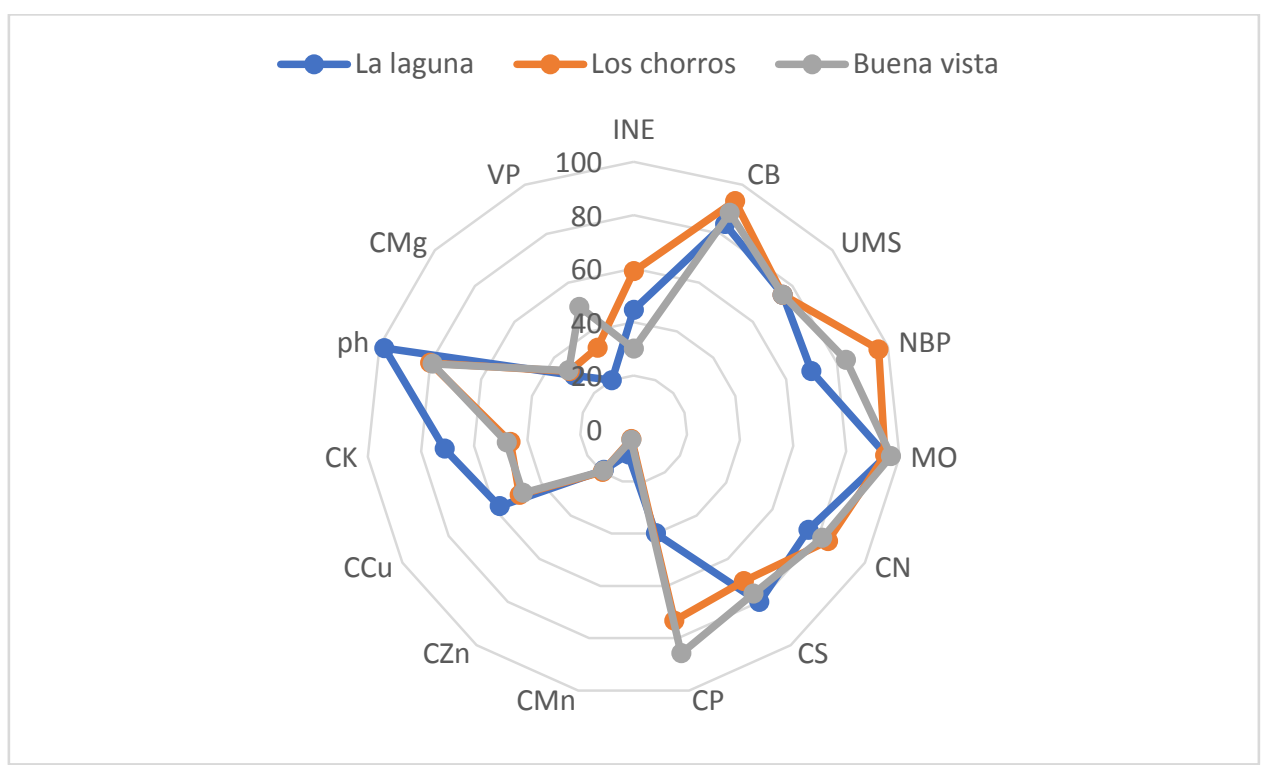

Fuente: elaboración propia.

Una alternativa de rentabilidad y aumento de producción es el uso de prácticas agrícolas amigables con el ambiente, las cuales buscan mejorar la estabilidad y fertilidad del suelo (Acevedo-Osorio et al., 2017). Como resultado, el desarrollo de cultivos no dependería en su totalidad de factores externos de mercado, tales como la inestabilidad de precios (Fonseca-Carreño et al., 2015). Avellaneda-Torrez et al. (2014) exponen un tipo de agricultura campesina en la que prima la seguridad alimentaria de la familia, alternativa no aceptada por los agricultores, debido a que se disminuiría la oferta de empleo y a que la diversificación de ingresos en el páramo no ofrece alternativas diferentes a la producción de papa, comercialización de leche y, en menor proporción, venta de carne ovina.

Para Acevedo-Osorio et al. (2017) y Barrezueta-Unda y Paz-González (2018), permitir la resiliencia social, a partir de la adaptación y disposición de los agricultores y entes gubernamentales para iniciar acciones colectivas a partir de la integración de conocimiento científico y local, contribuiría a disminuir los problemas asociados a bajas condiciones de equidad y bienestar social provocados en algunas fincas por la falta de capacitación y dignificación de la labor campesina. De ahí la importancia de promover estrategias de producción que generen empleos y nuevos ingresos, con la 
característica, en el caso de la papa, de no depender en su totalidad de insumos externos, manteniendo así la biodiversidad y su funcionalidad biológica y cultural (Bravo-Medina et al., 2017).

Estrategias como la producción en fincas con fundamentos agroecológicos, basados en el conocimiento de la flora nativa y las bondades del territorio en cuanto a riqueza biológica e hídrica permitirían, con la disposición técnica y financiera de las entidades competentes, acordar acciones de beneficio colectivo tales como reforestación, oferta de alternativas agropecuarias, saneamiento básico y ecoturismo (Avellaneda-Torres et al., 2014).

Finalmente, es pertinente mencionar que este trabajo es un diagnóstico inicial que depende de una continua evaluación de cada parámetro para optimizar los datos a partir de un monitoreo constante que permita identificar nuevos puntos críticos y ajustar las medidas de cada indicador y finca, con el fin de mejorar la construcción de estrategias basadas en la toma de decisiones concertadas entre entidades gubernamentales y los productores de papa de la región estudiada.

\section{CONCLUSIONES}

Los sistemas evaluados presentan similitudes en cuanto a técnicas de producción y uso de agroquímicos, situación que refleja la continua dependencia de insumos externos para priorizar el rendimiento de los cultivos de papa, dejando de lado la conservación del hábitat natural.

Las diferencias encontradas en los valores obtenidos de $\mathrm{N}, \mathrm{P}, \mathrm{Mg}, \mathrm{K}, \mathrm{Mn}$, el porcentaje de materia orgánica y el pH entre las fincas, la muestra del sitio no intervenido y los valores óptimos para la producción de papa según el laboratorio Agrosoil, sugieren la necesidad de asistencia técnica para usar los insumos en las concentraciones adecuadas, evitando así excesos en la concentración de nutrientes y, en consecuencia, alteraciones en la composición del suelo, además de daños ambientales.

\section{RECOMENDACIONES}

Es necesario acordar políticas públicas entre agricultores y entes gubernamentales con el fin de posibilitar acciones que permitan a la comunidad productora de papa consolidarse económica y socialmente, sin descuidar el uso adecuado de los recursos naturales.

Teniendo en cuenta la continua pérdida de biodiversidad en ecosistemas frágiles como el páramo, son necesarios estudios que evalúen el estado de su conservación, basados en el análisis de atributos en cuanto a composición, estructura y función. Estos resultados, integrados con estudios que especifiquen el bienestar social y la viabilidad económica, deben ser el centro de discusión para que organizaciones comunitarias, junto con entidades gubernamentales, acuerden acciones colectivas respecto a la adopción de sistemas de cultivo sustentables. 


\section{AGRADECIMIENTOS}

A los agricultores de la vereda Daita, en el municipio de Gámeza (Boyacá). A la Universidad Pedagógica y Tecnológica de Colombia, a través de la Vicerrectoría de Investigaciones y Extensión, y al Grupo de Investigación en Estudios de Etología, Ecología, Educación y Conservación (GECOS) por la convocatoria Joven Investigador, que permitió la financiación del proyecto con código SGI 2547.

\section{REFERENCIAS}

Acevedo-Osorio, A., Leiton, A. A., Durán, M. V. L., \& Quiroga, K. L. F. (2017). Sustentabilidad y variabilidad climática: Acciones agroecológicas participativas de adaptación y resiliencia socioecológica en la región alto-andina colombiana. Revista Luna Azul, 44, 6-26. https://doi.org/10.17151/luaz.2017.44.2

Álvarez-Yela, A. C., Alvarez-Silva, M. C., Restrepo, S., Husserl, J., Zambrano, M. M., Danies, G., \& González Barrios, A. F. (2017). Influence of agricultural activities in the structure and metabolic functionality of paramo soil samples in Colombia studied using a metagenomics analysis in dynamic state. Ecological Modelling, 351, 63-76. https://doi.org/10.1016/j.ecolmodel.2017.02.010

Antonini, C., \& Larrinaga, C. (2017). Planetary boundaries and sustainability indicators. A survey of corporate reporting boundaries. Sustainable Development, 137(1), 123-137. https://doi.org/10.1002/sd.1667

Avellaneda-Torres, L. M., Torres, E., \& León-Sicard, T. E. (2014). Agricultura y vida en el páramo: una mirada desde la vereda El Bosque (Parque Nacional Natural de Los Nevados). Cuadernos de Desarrollo Rural, 11(73), 105-128. https://doi.org/10.11144/Javeriana.CDR11-73.avpm

Avellaneda-Torres, L. M., Torres, E., \& León-Sicard, T. E. (2015). Alternativas ante el conflicto entre autoridades ambientales y habitantes de áreas protegidas en páramos colombianos. Mundo Agrario, 16(31), 1-27. http://www.memoria.fahce.unlp.edu.ar/art_revistas/pr.6742/pr.6742.pdf

Avellaneda-Torres, L. M., León-Sicard, T. E., \& Torres, E. (2018). Impact of potato cultivation and cattle farming on physicochemical parameters and enzymatic activities of Neotropical high Andean Páramo ecosystem soils. Science of the Total Environment, 631, 1600-1610. https://doi.org/10.1016/j.scitotenv.2018.03.137

Barrezueta-Unda, S., \& Paz-González, A. (2018). Indicadores de sostenibilidad sociales y económicos : Caso productores de cacao en El Oro, Ecuador. Revista Ciencia UNEMI, 11(27), 20-29. https://doi.org/10.29076/issn.2528-7737vol11iss27.2018pp20$29 p$

Bonisoli, L., Galdeano-Gómez, E., \& Piedra-Muñoz, L. (2018). Deconstructing criteria and assessment tools to build agri-sustainability indicators and support farmers' decision-making process. Journal of Cleaner Production, 182, 1080-1094. https://doi.org/10.1016/j.jclepro.2018.02.055 
Bravo-Medina, C., Marín, H., Marreno-Labrador, P., Ruiz, M., TorresNavarrete, B., Navarrete-Alvarado, H., \& Changoluisa-Vargas, D. (2017). Evaluación de la sustentabilidad mediante indicadores en unidades de producción de la provincia de Napo, Amazonia ecuatoriana. Bioagro, 29(1), 23-36. http://ve.scielo.org/pdf/ba/v29n1/art03.pdf

Calleros-Islas, A. (2019). Sustainability assessment. An adaptive low-input tool applied to the management of agroecosystems in México. Ecological Indicators, 105, 386-397. https://doi.org/10.1016/j.ecolind.2017.12.040

Cárdenas, G. I., \& Acevedo, A. (2015). Evaluación de la sustentabilidad de los sistemas productivos campesinos de la Asociacion de Caficultores Orgánicos de Colombia -ACOC-, Valle del Cauca. Producción Agropecuaria y Desarrollo Sostenible, 4(1), 109-135. https://doi.org/10.5377/payds.v4i0.3967

Cruz-Mendoza, J., Villegas-Aparicio, Y., Jerez-Salas, M. P., Pérez-León, M. J., Vinay-Vadillo, J. C., \& Castañeda-Hidalgo, E. (2016). Sustentabilidad de tres sistemas de producción ovina en los valles centrales de Oaxaca. Revista Mexicana de Agroecosistemas, 3(1), 49-60. https://www.voaxaca.tecnm.mx/revista/docs/RMAE\%20vol\%203_1_2016/5\%20RMA E-2016-01-Ovinos-Galeras.pdf

Erbaugh, J., Bierbaum, R., Castilleja, G., Da-Fonseca, A. B., \& Hansen, C. B. (2019). Toward sustainable agriculture in the tropics. World Development, 121, 158162. https://doi.org/10.1016/j.worlddev.2019.05.002

Feinsinger, P. (2003). El diseño de estudios de campo para la conservación de la biodiversidad. Editorial FAN.

Fonseca-Carreño, J. A., Cleves-Leguízamo, J. A., \& León-Sicard, T. E. (2015). Evaluación de la sustentabilidad de agroecosistemas familiares campesinos en la microcuenca del río Cormechoque (Boyacá). Revista Ciencia y Agricultura, 13(1), 2947. https://doi.org/10.19053/01228420.4804

Gitari, H. I., Gachene, K. K., Karanja, N. N., Kamau, S., Nyawade, S., Sharma, K., \& Schulte-Geldermann, E. (2018). Optimizing yield and economic returns of rainfed potato (Solanum tuberosum L.) through water conservation under potato-legume intercropping systems. Agricultural Water Management, 208, 59-66. https://doi.org/10.1016/j.agwat.2018.06.005

Insuasty-Córdoba, S. C., Ramos-Zambrano, H. S., Marcillo-Paguay, C. A., López-Peñafiel, H. V., Mateus-Rodríguez, J. F., \& Martínez-Pachón, E. (2020). Diagnóstico financiero y biofísico para la producción de semilla de papa. Agronomía Mesoamericana, 31(3), 628-640. https://doi.org/10.15517/am.v31i3.39940

Incacari, C., Otiniano, A. J., Lastarria, R. C., \& Cortez, J. M. (2019). Sustentabilidad de las unidades productoras de papa (Solanum tuberosum L.), en Jauja, Perú. Revista Ciencia e Investigación, 4(2), 21-27. https://doi.org/10.5281/zenodo.3240674

Masera, O., Astier, M., \& López-Ridaura, S. (2000). Sustentabilidad y manejo de recursos naturales. El marco de evalaución MESMIS. Mundi-Prensa México, S.A. 
Márquez, L. E., Cuétara-Sánchez, L., Bernardo-Vélez, J., \& Mera-Ponce, D. (2020). Sistema de indicadores para la evaluación de la sostenibilidad económica del sector hotelero en la parroquia Crucita, Manabí, Ecuador. Espacios, 41(03), 3-13.

Montes-Pérez, R., Ceballos, A., Novelo, L., Palma, I., Magaña, J., \& Sierra, Á. (2016). Evaluación de la sustentabilidad de dos unidades de producción ovina en Yucatán, México. Abanico Veterinario, 6(2), 39-53. http://dx.doi.org/10.21929/abavet2016.62.4

Motta-Delgado, P. A., Ocaña Martínez, E. H., \& Rojas-Vargas, E. P. (2019). Indicadores asociados a la sostenibilidad de pasturas: una revisión. Ciencia y Tecnología Agropecuaria, 20(2), 387-408. https://doi.org/10.21930/rcta.vol20_num2_art:1464

Tang, J., Wang, J., Fang, Q., Dayananda, B., Yu, Q., Zhao, P., \& Pan, X. (2019). Identifying agronomic options for better potato production and conserving water resources in the agro-pastoral ecotone in North China. Agricultural and Forest Meteorology, 272, 91-101. https://doi.org/10.1016/j.agrformet.2019.04.001

Van-Dingenen, J., Hanzalova, K., Abd-Allah, M., Abel, C., Seibert, T., Giavalisco, P., \& Wahl, V. (2019). Limited nitrogen availability has cultivar-dependent effects on potato tuber yield and tuber quality traits. Food Chemistry, 288, 170-177. https://doi.org/10.1016/j.foodchem.2019.02.113 